\title{
Dynamic behavior of nematic liquid crystal mixtures with quantum dots in electric fields
}

\author{
Emil Petrescu, Cristina Cirtoaje ${ }^{*}$ and Octavian Danila
}

\author{
Full Research Paper \\ Address: \\ University Politehnica of Bucharest, Department of Physics, Splaiul \\ Independenței 313, 060042, Bucharest, Romania \\ Email: \\ Cristina Cirtoaje ${ }^{*}$ - cristina.cirtoaje@upb.ro \\ * Corresponding author \\ Keywords: \\ Fréedericksz transition; nematic liquid crystals; quantum dots
}

Beilstein J. Nanotechnol. 2018, 9, 399-406.

doi:10.3762/bjnano.9.39

Received: 26 July 2017

Accepted: 04 January 2018

Published: 01 February 2018

This article is part of the Thematic Series "Nanostructured liquid crystal systems and applications".

Guest Editor: A. Emelyanenko

(C) 2018 Petrescu et al.; licensee Beilstein-Institut.

License and terms: see end of document.

\begin{abstract}
The dynamic behavior of a mixture consisting of liquid crystalline 4-cyano-4'-pentylbiphenyl (5CB) and CdSe/ZnS quantum dots in electric fields was theoretically studied. The model was based on elastic continuum theory considering the interaction of the nematic molecules with the surrounding molecules, with the quantum dots and with the electric field. Experimental data obtained by dynamic measurements on a sample containing $0.89 \%$ (mass fraction) of $\mathrm{CdSe} / \mathrm{ZnS}$ quantum dots revealed a decrease of the relaxation time compared to pure $5 \mathrm{CB}$.
\end{abstract}

\section{Introduction}

The expansion of liquid crystal (LC)-based devices in common life domains as well as science and engineering, requires improved technologies and new materials. Materials science provides great opportunities to these technologies by synthesizing new nanoparticles that can be mixed with liquid crystals: carbon nanotubes [1-3], graphene, magnetic nanoparticles [4,5], gold nanoparticles [6], quantum dots (QDs) [7-12] or other nanomaterials [13-17] that can be effectively used in electrooptical devices.

When added to liquid crystals, quantum dots may seriously influence their behavior under an electric field due to the anchoring forces acting on the quantum dot surface. Dynamic experiments performed in alternating electric fields proved that by adding a small amount of $\mathrm{CdSe} / \mathrm{ZnS}$ quantum dots in thermotropic nematic liquid crystal with positive dielectric anisotropy, we obtain a decrease of the relaxation time.

When an external electric field higher than the Fréedericksz transition threshold $[18,19]$ is applied transversely to a planar liquid crystal cell, the molecules change their orientation tending to align their director in parallel to the field. This reorientation is illustrated by an intensity variation of a laser beam crossing through the sample. The time period after which a con- 
stant intensity is obtained, which means that an equilibrium state is reached, is characterized by the relaxation time and can be experimentally evaluated from plotting the intensity as a function of the time. A theoretical analysis of the LC + QDs system based on elastic continuum theory allowed us to express the time evolution of the molecular distortion angle inside the cell and to calculate the relaxation time. Theoretical values and experimental data were in good agreement showing that the model offers an important tool for engineers to precisely control LC-based device parameters.

\section{Theoretical Background}

When a nematic liquid crystal with positive dielectric anisotropy is exposed to an electric field, and if the intensity of the field is higher than the Fréedericksz transition threshold, the molecular director changes its orientation, trying to align in parallel with the field direction. During this reorientation process, the refractive index of the sample also changes:

$$
\frac{1}{n_{\mathrm{eff}}^{2}}=\frac{\sin ^{2} \theta}{n_{\mathrm{o}}^{2}}+\frac{\cos ^{2} \theta}{n_{\mathrm{e}}^{2}}
$$

where $n_{\mathrm{o}}$ and $n_{\mathrm{e}}$ are, respectively, the ordinary and extraordinary refractive indexes and $\theta$ is the angle between the direction of the light and $n_{\mathrm{e}}$. Between the extraordinary and ordinary rays, there is a path difference which can be calculated as a function of the cell thickness, $L$ :

$$
\delta=\int_{-L / 2}^{L / 2}\left(n_{\mathrm{eff}}-n_{\mathrm{o}}\right) \mathrm{d} z
$$

with the corresponding phase difference $\Delta \varphi=(2 \pi \delta) / \lambda$.

For a planar cell configuration we have:

$$
\theta=\theta_{\mathrm{m}} \cos \frac{\pi z}{L}
$$

where $\theta_{\mathrm{m}}$ is the maximum deviation angle (in the middle in the cell). If small deviation angles are considered, the phase difference becomes:

$$
\Delta \varphi=\frac{\pi L \Delta n}{2 \lambda}\left[2-\theta_{\mathrm{m}}^{2}+\frac{\theta_{\mathrm{m}}^{4}}{4}\right]
$$

and the intensity of the laser beam crossing through the sample is:

$$
I=I_{0} \sin ^{2}\left[\frac{\pi L \Delta n}{2 \lambda}\left(2-\theta_{\mathrm{m}}^{2}+\frac{\theta_{\mathrm{m}}^{4}}{4}\right)\right]
$$

The equation of the maximum deviation angle as a function of time is obtained from the total free energy of the system by applying the Euler-Lagrange equation. Elastic continuum theory states that a liquid crystal is acting like a continuous fluid and the interaction forces between its molecules are elastic. Taking into account a strong anchoring on the glass support, the free energy density of such a system with added quantum dots is:

$$
f=f_{N}+f_{E}+f_{I N T},
$$

where $f_{N}$ is the liquid crystal free energy density, $f_{E}$ is a term combining the electric field action on the liquid crystal molecules in the presence of quantum dots and $f_{I N T}$ reflects the interaction between quantum dots and liquid crystal molecules.

For a planar aligned cell (Figure 1) with thickness $L$, and when the voltage is just above the Fréedericksz transition threshold, the deviation angle is very small and the free energy caused by the elastic forces is:

$$
f_{N}=\frac{1}{2}\left(K_{1} \cos ^{2} \theta+K_{3} \sin ^{2} \theta\right) \theta_{z}^{2}
$$

where $K_{1}$ and $K_{3}$ are the splay and bent elastic constants and $\theta_{z}=\partial \theta / \partial z$.

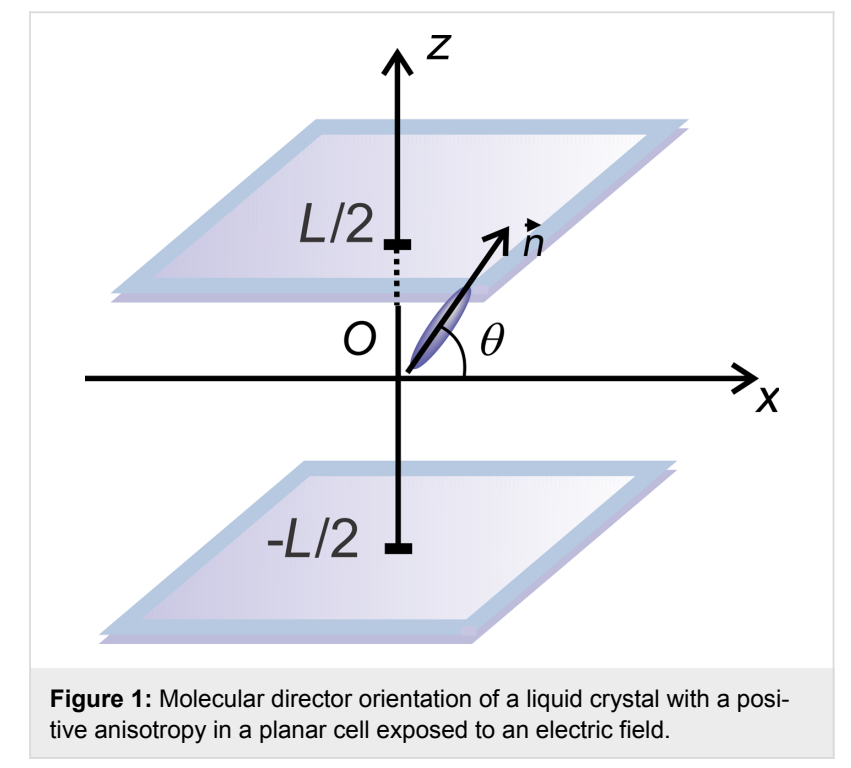

Due to the presence of the QDs, the electric properties of the mixture change. Hence, instead of perpendicular dielectric 
permittivity, $\varepsilon_{\perp}$, and electric anisotropy, $\varepsilon_{a}$, we use the effective perpendicular permittivity, $\varepsilon_{\perp e f f}$, and effective anisotropy, $\varepsilon_{\text {aeff }}$.

$$
f_{E}=-\frac{1}{2} \varepsilon_{0}\left[\varepsilon_{\perp e f f} E^{2}+\varepsilon_{\text {aeff }}(\vec{n} \vec{E})^{2}\right] .
$$

If a small spherical cavity is considered inside the liquid crystal, a polarisation field on the hole surface appears. According to classical theory developed by Landau and Lifshitz [20], the polarization field around a spherical cavity inside a dielectric medium is proportional to the applied field, so:

$$
E_{Q D}=H E .
$$

Inside an anisotropic dielectric (the liquid crystal) the spherical cavity changes into an ellipsoid with the semi-axes $b / \sqrt{1 / \varepsilon_{\|}}$ and $b \sqrt{1 / \varepsilon_{\perp}}$, where $b$ is the cavity radius, $\varepsilon_{\|}$is the parallel permittivity, $\varepsilon_{\perp}$ is the perpendicular permittivity. In this case $H$ becomes:

$$
H=\frac{1 / \varepsilon_{\|}}{1 / \varepsilon_{\|}-d(z)\left(1 / \varepsilon_{\|}-1\right)}
$$

where $d(z)$ the depolarization coefficient, which for an ellipsoid with almost spherical shape (horizontal axis equal $b=R$ and vertical axis $a=R+0.0005 R)$ is

$$
d(z)=\frac{1}{3}-\frac{2}{15}\left(1-\frac{b}{a}\right)
$$

If a quantum dot is placed inside this cavity, another polarization field will occur but because of its semiconductor nature, this field is much smaller than the one produced at the surface of the dielectric and might be neglected. Thus, the effective field acting on the nematic molecules is smaller than the applied field $E$ by $E_{Q D}$, i.e., $E^{\prime}=E-H E$.

The free energy density may also be written as:

$$
f_{E}=-\frac{1}{2} D E^{\prime}=-\frac{1}{2} D(1-H) E
$$

where $D$ is the electric induction. Thus the free energy is:

$$
F_{E}=\int_{-L / 2}^{L / 2} f_{E} \mathrm{~d} z=-\frac{D}{2}(1-H) \int_{-L / 2}^{L / 2} E \mathrm{~d} z
$$

By taking into account that the applied voltage on the sample is

$$
U=\int_{-L / 2}^{L / 2} E \mathrm{~d} z
$$

and considering small angle approximation for the configuration presented in Figure 2 we get:

$$
\begin{aligned}
F_{E} & =\int_{-L / 2}^{L / 2}\left[-\frac{\varepsilon_{0} \varepsilon_{\perp e f f}(1-H) U^{2}}{2 L^{2}}\right. \\
& \left.-\frac{\varepsilon_{0} \varepsilon_{\text {aeff }}(1-H) U^{2}}{2 L^{2}} \sin \theta^{2}\right] \mathrm{d} z .
\end{aligned}
$$

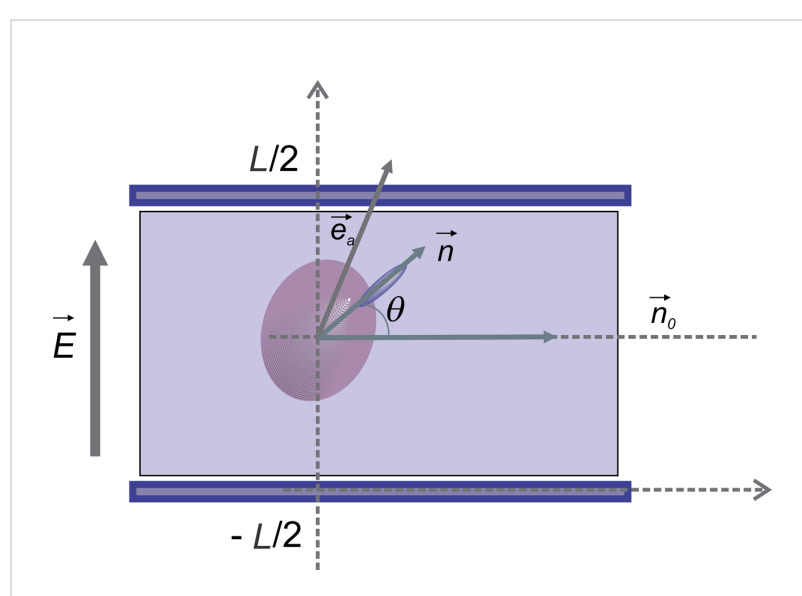

Figure 2: Molecular director and quantum dot orientation in a planar LC cell subjected to electric field.

Then the free energy density is:

$f_{E}=-\frac{\varepsilon_{0} \varepsilon_{\perp e f f}(1-H) U^{2}}{2 L^{2}}-\frac{\varepsilon_{0} \varepsilon_{a e f f}(1-H) U^{2}}{2 L^{2}} \sin \theta^{2}$

The first term does not depend on the molecular orientation so we may neglect it because it disappears when applying the Euler-Lagrange equation. Thus, we may write:

$$
f_{E}=-f_{0 E} \sin ^{2} \theta
$$

where

$$
f_{0 E}=\frac{\varepsilon_{0} \varepsilon_{a e f f}(1-H) U^{2}}{2 L^{2}}
$$


and

$$
\varepsilon_{a e f f}=\varepsilon_{a}+c_{v} \frac{3\left(\varepsilon_{Q D}-\varepsilon_{a}\right) \varepsilon_{a}}{\varepsilon_{Q D}+2 \varepsilon_{a}}
$$

is the effective dielectric anisotropy of the liquid crystal containing quantum dots as a function of the dielectric anisotropy of the LC, $\left(\varepsilon_{a}\right)$, of the dielectric constant of the QDs $\left(\varepsilon_{Q D}\right)$ and the volumetric fraction of the quantum dots $\left(c_{v}\right)$.

The intraction free energy between nematic molecules and a quantum dot can be calculated from the Rapini formula [21] Considering an elliptical quantum dot with one of the axes just a little bit longer than the others and the configuration given in Figure 3, as well as homeotropic anchoring of liquid crystal molecules on the QD surface [12], the interaction energy for a particle can be obtained using a similar procedure as presented by Burylov [22]:

$$
f_{p}=\frac{w}{2} \int\left|\vec{n}_{0 s} \times \vec{n}_{0}\right|^{2} \mathrm{~d} S,
$$

where $w$ is the anchoring energy on the surface of the nanoparticle, $\vec{n}_{0 s}$ is the nematic director on the QD surface and $\vec{n}_{0}$ is the direction of molecular director far away from the QD surface.

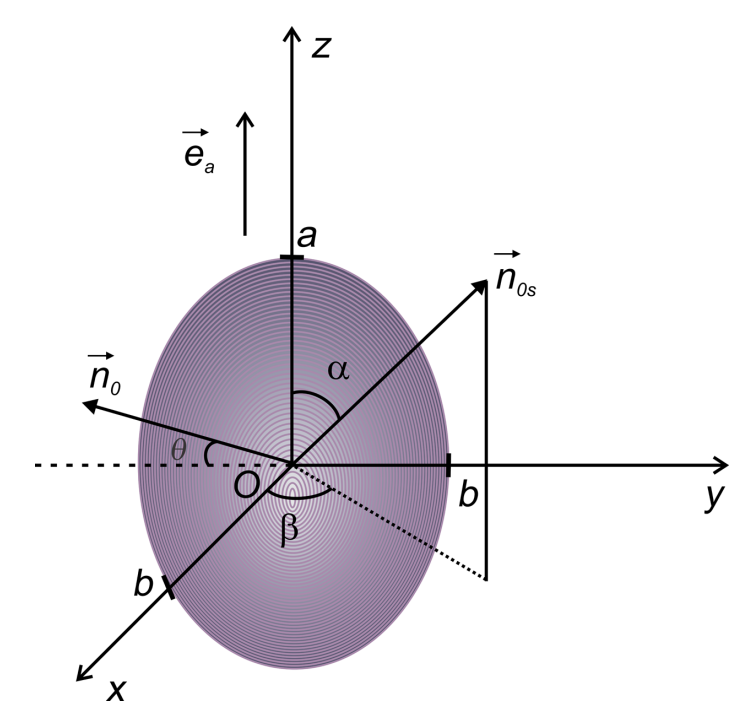

Figure 3: Nematic molecular orientation around an ellipsoidal QD

According to the manufacturers, the quantum dots are spherical particle with the radius $R=3 \mathrm{~nm}$ (6 $\mathrm{nm}$ in diameter). Considering their small anisotropy we approximate the sphere with an almost spherical ellipsoid having a short axis $(b=R)$ and a long axis close to $b$. Thus, we obtain:

$$
\begin{aligned}
f_{p} & =\frac{w b^{2}}{2} \int_{\alpha, \beta}\left\{\left[\sin ^{2} \alpha \cdot \sin ^{2} \beta\right.\right. \\
& \left.+(\sin \alpha \cos \beta \cos \theta-\cos \alpha \cdot \sin \theta)^{2}\right] \\
& \left.\times \sin \alpha \sqrt{\frac{a}{b} \cos ^{2} \alpha+\sin ^{2} \alpha}\right\} \mathrm{d} \beta \mathrm{d} \alpha,
\end{aligned}
$$

This equation can be solved and written in a simplified form as:

$$
f_{p}=f_{p c t}+f_{p 0} \cos ^{2} \theta
$$

where $f_{p c t}$ is a constant depending on the anchoring energy and the particle geometry and disappears when applying the Euler-Lagrange equations. Thus, it may be neglected and $f_{p 0}=11.52 w b^{2} \pi$. Considering the volumetric concentration of quantum dots, we obtain:

$$
f_{I N T}=\frac{3 f_{0} c_{v}}{2 \pi R^{3}} \sin ^{2} \theta
$$

where $f_{0}=(11.52 / 2) w \pi R^{2}$ is a constant depending on the geometrical parameters of the system and the LC + QD interaction energy calculated for the ellipsoid QD presented in Figure 3. When a dynamic study of the mixture is made, the total free energy density must also take into account the dissipative time-dependent term, and the total free energy becomes:

$$
\begin{aligned}
f & =\frac{1}{2}\left(K_{1} \cos ^{2} \theta+K_{3} \sin ^{2} \theta\right) \theta_{z}^{2} \\
& -f_{0 E} \sin ^{2} \theta+\frac{3 f_{0} c_{v}}{2 \pi R^{3}} \sin ^{2} \theta-\frac{1}{2} \gamma\left(\frac{\partial \theta}{\partial t}\right)^{2} .
\end{aligned}
$$

By applying the Euler-Lagrange equation and assuming that $\theta$ is small we obtain:

$$
\begin{aligned}
& {\left[K_{1}\left(1-\theta^{2}\right)+K_{3}\right] \theta_{z z}+\left(K_{3}-K_{1}\right) \theta \theta_{z}^{2}} \\
& +2\left(f_{0 E}-\frac{3 f_{0} c_{v}}{2 \pi R^{3}}\right)\left(\theta-\frac{2 \theta^{3}}{3}\right)=\gamma \frac{\partial \theta}{\partial t},
\end{aligned}
$$

where $\theta_{z z}=\partial^{2} \theta / \partial z^{2}$. Considering the deviation angle from Equation 3, we obtain the dependency of the deviation angle on the relaxation time: 


$$
-\theta_{m}^{2}+\alpha \theta_{m}^{4}=\frac{\gamma}{2\left(\frac{K_{1} \pi^{2}}{L^{2}}+2 C\right)} \frac{\mathrm{d} \theta_{m}^{2}}{\mathrm{~d} t},
$$

where

$$
\alpha=\frac{C-\frac{\pi^{2}}{2 L^{2}}\left(K_{3}-K_{1}\right)}{\frac{K_{1} \pi^{2}}{L^{2}}+2 C}
$$

and

$$
C=\frac{3 f_{0} c_{v}}{\pi^{2} R^{3}}-f_{0 E}
$$

Solving this equation leads to

$$
\theta_{\mathrm{m}}^{2}(t)=\frac{\theta_{\mathrm{m}}^{2}(\infty)}{1+\left[\frac{\theta_{\mathrm{m}}^{2}(\infty)}{\theta_{\mathrm{m}}^{2}(0)}-1\right] \exp \left(-\frac{t}{\tau_{\text {on }}}\right)},
$$

where $\theta_{\mathrm{m}}(\infty)$ and $\theta_{\mathrm{m}}(0)$ are the limit values of the deviation angle in the center of the cell and $\tau_{o n}$ is:

$$
\tau_{o n}=\frac{\gamma L^{2}}{2 \pi^{2} K_{1}\left[\frac{L^{2}}{K_{1} \pi^{2}}\left(2 f_{0 E}-\frac{3 f_{0} \gamma L^{2} c_{v}}{\pi R^{3}}\right)-1\right]} .
$$

When the voltage is switched off, we follow the same procedure after replacing $f_{O E}=0$ in Equation 22. Thus, we obtain:

$$
\theta_{\mathrm{m}}^{2}(t)=\frac{\theta_{\mathrm{m}}^{2}(0)}{\xi \theta_{\mathrm{m}}^{2}(0)+\left[1-\xi \theta_{\mathrm{m}}^{2}(0)\right] \exp \left(\frac{t}{\tau_{o f f}}\right)},
$$

where $\theta_{\mathrm{m}}(0)$ is the maximum deviation angle at $t=0, \xi$ is a constant depending on LC properties and $\tau_{\text {off }}$ is:

$$
\tau_{o f f}=\frac{\gamma L^{2}}{2 \pi^{2} K_{1}\left[1+\frac{3 f_{0} c_{v}}{2 \pi R^{3}}\right]} .
$$

\section{Experimental}

For the sample preparation we used $\mathrm{CdSe} / \mathrm{ZnS}$ quantum dots in toluene with an average size of $6 \mathrm{~nm}$ and a fluorescence wavelength of $630 \mathrm{~nm}$ from Aldrich. The QD suspension was left in a bottle in clean vacuum environment until the toluene was completely evaporated. Then, 4-cyano-4'-pentylbiphenyl (5CB) was added and sonicated for three days, several hours each day until a homogenous pink mixture was obtained. The homogeneity was checked by using polarization microscopy. The mass concentration was $0.89 \%$, which led to a volumetric fraction of $1.16 \%$ (the QD density is $0.87 \mathrm{~g} / \mathrm{cm}^{3}$ and the density of $5 \mathrm{CB}$ is $1.02 \mathrm{~g} / \mathrm{cm}^{3}$ ). The mixture was used to fill a $15 \mu \mathrm{m}$ thick planar aligned cell from Instek, which was mounted in a holder placed in a Mettler Toledo hot stage. A reference sample was made using pure $5 \mathrm{CB}$. The terminals of the holder were connected to a power source that produced a square wave signal with a frequency of $10 \mathrm{kHz}$. For voltages higher than the Fréedericksz transition threshold, the nematic director changes its orientation trying to align with the field. A He-Ne laser beam crossed the sample through the windows of the holder and the emergent signal was recorded with a Thor Lab photovoltaic cell. Two crossed polarizers at $45^{\circ}$ were placed on both sides of the sample to obtain equal intensities for ordinary and extraordinary rays. An acquisition system connected to a computer allowed data recording every $20 \mathrm{~ms}$. A schematic representation of the experimental setup is presented in Figure 4.

\section{Results and Discussion}

Fréedericksz transition threshold and relaxation time were determined for both samples at a constant temperature of $28^{\circ} \mathrm{C}$. The Fréedericksz transition threshold was determined by slowly increasing the applied voltage and while recording the laser in-

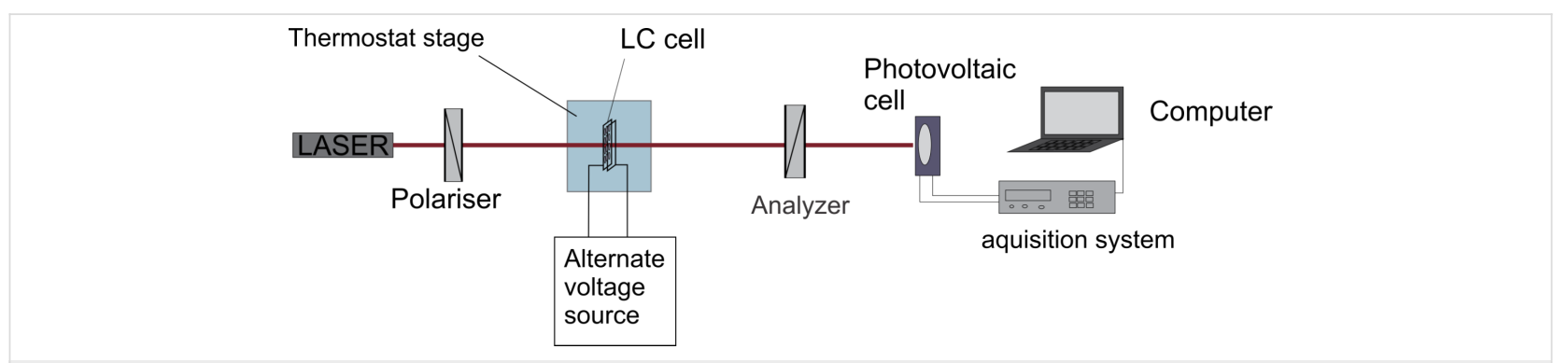

Figure 4: Experimental setup for the dynamic study of the LC + QDs mixture. 
tensity. Preliminary measurements showed no changes of the laser intensity below $0.5 \mathrm{~V}$. Hence, the voltage steps were $0.1 \mathrm{~V}$ below this value and $0.01 \mathrm{~V}$ above it. The data were represented in an intensity versus voltage plot (Figure 5). Above the threshold value, the intensity of the emergent laser beam exhibits a series of maxima and minima.

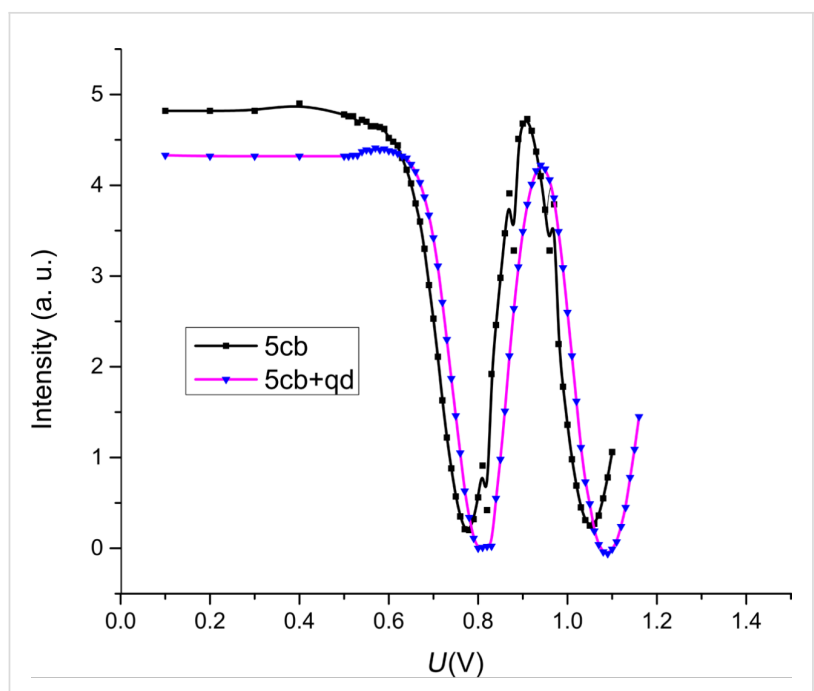

Figure 5: Fréedericksz transition for $5 \mathrm{CB}$ and $5 \mathrm{CB}+\mathrm{QD}$. Lines are guide to the eyes.

For the dynamic measurements the value of the first intensity maximum was chosen. Thus, the applied voltage was without any doubt above the Fréedericksz transition and the deviation angle was small as considered in the theoretical model. The intensity versus time plot was recorded for each sample and the function in Equation 5 was used to fit the data. A power switch was connected to the acquisition system so that when the field was switched on the acquisition started at the same time to measure the intensity when the field is switched on. A similar procedure was used when the power source was disconnected and at the same time the acquisition started to measure intensity versus time plot when the field was switched off.

In Figure 6 we presented the light intensity versus time plot for the pure LC (Figure 6a,b) and for the mixture of LC with QDs (Figure 6c,d). Experimental data were fitted using Equation 5 with the time dependency of $t$ thet $a_{\mathrm{m}}$ given by Equation 25 and Equation 27, where $L=15 \mu \mathrm{m}, K_{1}=6.2 \times 10^{-12} \mathrm{~N}, R=3 \mathrm{~nm}$ and $c_{v}=1.16 \%$ (volumetric fraction). Therefore, we obtain for the sample containing QDs, the experimental relaxation time $\tau_{\text {on-exp }}=0.167 \mathrm{~s}$ when the field is switched on and $\tau_{\text {off-exp }}=0.139 \mathrm{~s}$ when the field is switched off.

By fitting the data of the $5 \mathrm{CB}$ sample, we obtained a relaxation time $\tau_{\text {on-exp } 0}=0.172 \mathrm{~s}$ when the field is switched on and $\tau_{\text {off-exp } 0}=0.157 \mathrm{~s}$ when the field is switched off. The relaxation time decreases when quantum dots are added. Generally, this result was reported in similar researches [9-11] but only when the field was switched on. When the field was off the results in [9] showed an increase of the relaxation time for the sample containing quantum dots. The measurements were made on similar systems both of which emplyed $\mathrm{CdSe} / \mathrm{ZnS}$ in $5 \mathrm{CB}$ but in our case a temperature of $28{ }^{\circ} \mathrm{C}$ yielded a lower viscosity and, comsequently, a lower relaxation time especially when the field is switched off. In [8] the authors demonstrate an increase of the elastic coefficient $K_{1}$ with the QD concentration, which is in good agreement with Equation 26 and Equation 28 where the effective elastic constant is proportional with the volumetric fraction of the added quantum dots.

Basu and co-workers [7] proposed a different model of a quantum dot dispersion in a nematic liquid crystal. According to this model, dots are gathering together forming long chains around which liquid crystal molecules are homogeneously aligned. Such an alignment could not explain the decrease of the relaxation time when the field is switched on because the anchoring forces on quantum dots surface will slow down the molecular movement.

A possible explanation for this behavior might be given considering a homeotropic alignment of the LC molecules on the QD surface [12]. Thus, if the applied field is high enough to exceed the Fréedericksz transition threshold some of the molecules are already parallel to the field and only those placed on the horizontal sphere circumference are parallel to the cell plates, so the response time is shorter in the quantum dots sample (Figure 7a). When the field is switched off, all the molecules are distorted and their returning to the original position is "helped" by the anchoring forces on the QD surface and a slight decrease of the relaxation time is observed (Figure $7 b$ ).

\section{Conclusion}

We observed an improvement in the turn-off time of the nematic liquid crystal $5 \mathrm{CB}$ containing $\mathrm{CdSe} / \mathrm{ZnS}$ quantum dots of a nominal size of $6 \mathrm{~nm}$ diameter. The results are potentially useful in other cell geometries and with other materials. The theoretical model might be extended to other geometries and used to theoretically optimize the performance of QD-liquid crystal composites. The calculations presented in this paper are based on particular experimental results obtained at $28{ }^{\circ} \mathrm{C}$, for a relatively high concentration of quantum dots. Further measurements are to be done in the future, over a wide temperature range and under consideration of other parameters, to improve this model and obtain an accurate theoretical description of these systems. 


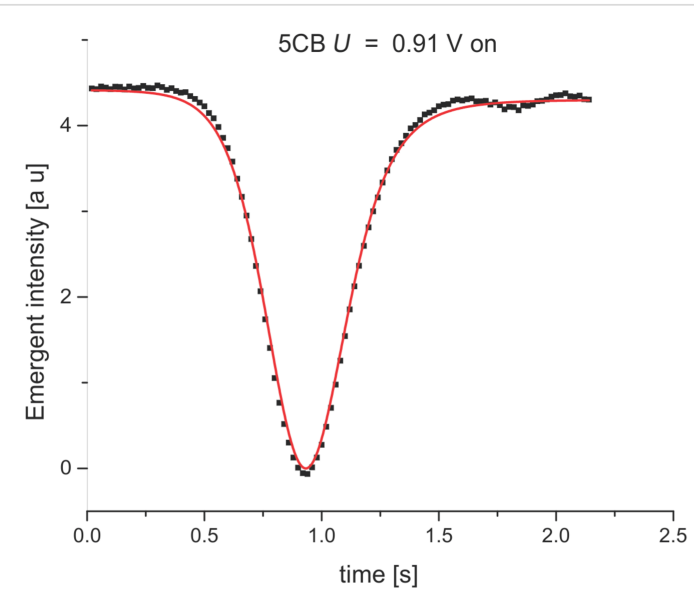

a)

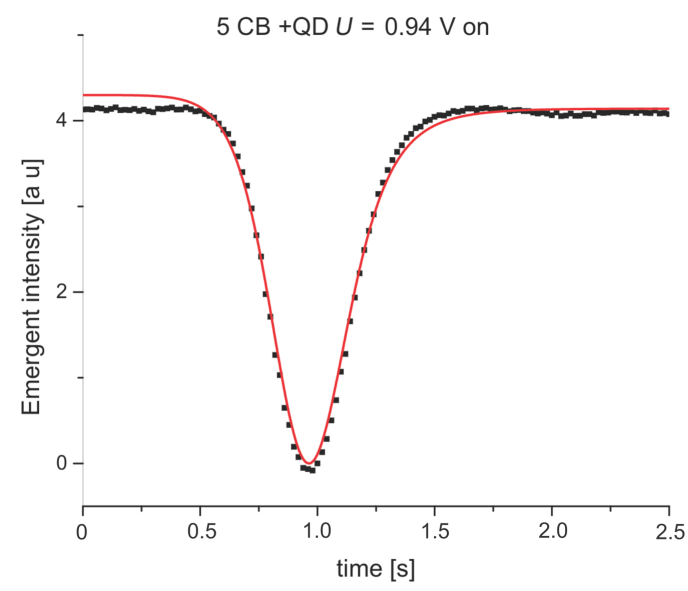

c)

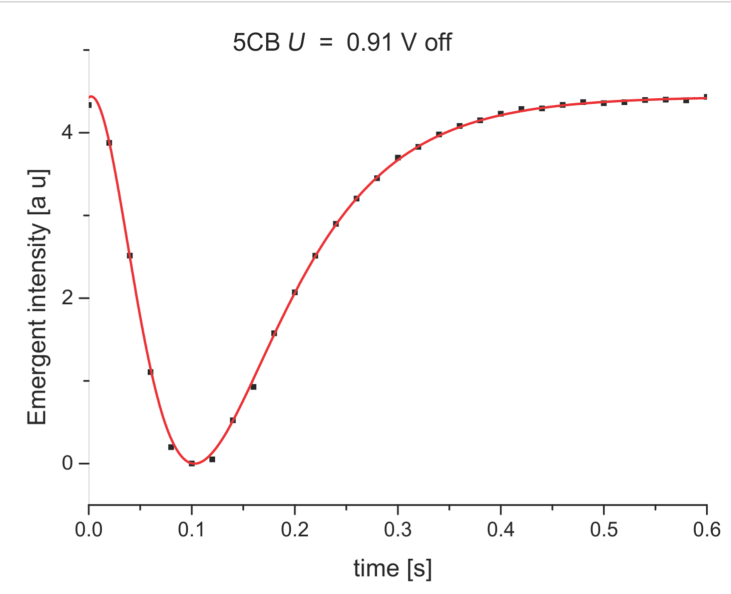

b)

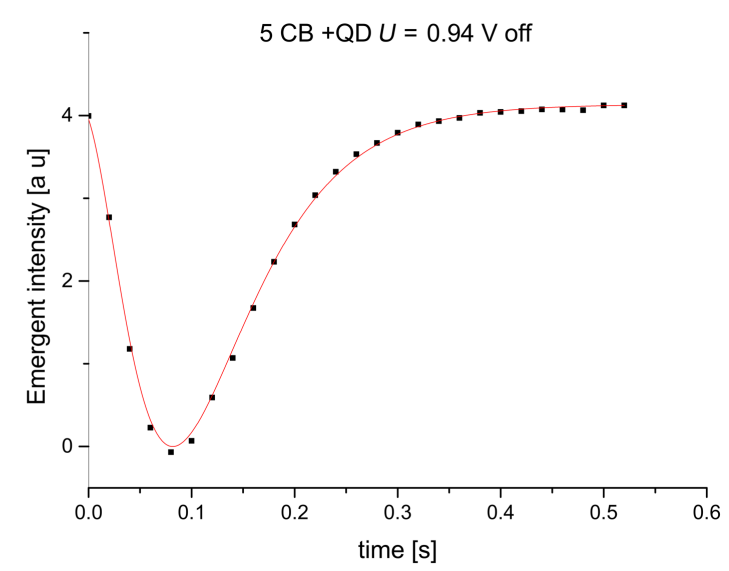

d)

Figure 6: Dynamic measurement of emergent light intensity: a) for LC sample when the field is on; b)for LC sample when the field is off; c) for LC + QDs sample when the field is on; for LC + QDs sample when the field is off. The dots represent experimental values and the red lines are theoretical representations.

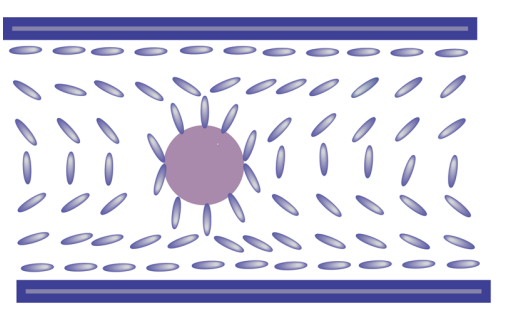

a)

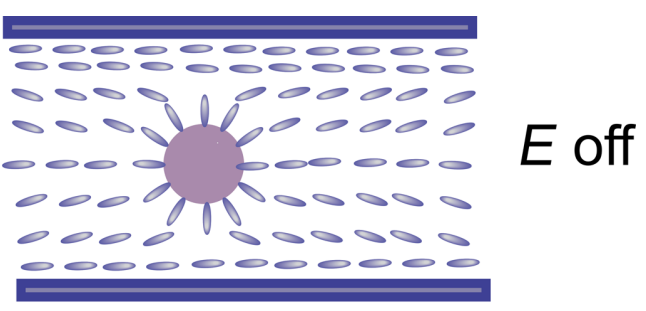

b)

Figure 7: Schematic representation of the molecular orientation around a QD a) when the field is switched on b) when the field is switched off.

\section{Acknowledgements}

The work has been funded by University Politehnica of Bucharest, through the 'Excellence Research Grants' Program,
UPB-GEX. Identifier: UPB-EXCELENȚ Ă-2016 Research project title Phase modulation in nematic liquid crystals subjected to magnetic field. Contract number 24/26.09.2016. 


\section{References}

1. Cirtoaje, C.; Petrescu, E. Physica E 2016, 84, 244-248. doi:10.1016/j.physe.2016.06.011

2. Samoilov, A. N.; Minenko, S. S.; Fedoryako, A. P.; Lisetski, L. N.; Lebovka, N. I.; Soskin, M. S. Funct. Mater. 2014, 21, 190-194. doi:10.15407/fm21.02.190

3. Bale, S.; Liyana-Arachchi, T. P.; Hung, F. R. Mol. Simul. 2016, 42, 1242-1248. doi:10.1080/08927022.2016.1174859

4. Cirtoaje, C.; Petrescu, E.; Stan, C.; Creanga, D. Physica E 2016, 79, 38-43. doi:10.1016/j.physe.2015.12.006

5. Mănăilă-Maximean, D.; Cîrtoaje, C.; Dănilă, O.; Donescu, D. J. Magn. Magn. Mater. 2017, 438, 132-137. doi:10.1016/j.jmmm.2017.02.034

6. Lewandowski, W.; Fruhnert, M.; Mieczkowski, J.; Rockstuhl, C.; Górecka, E. Nat. Commun. 2015, 6, 6590. doi:10.1038/ncomms7590

7. Basu, R.; lannacchione, G. S. Phys. Rev. E 2009, 80, 010701. doi:10.1103/PhysRevE.80.010701

8. Vakulin, D. A.; Frenkel, D. A.; Gavrish, E. O.; Konshina, E. A. Mol. Cryst. Liq. Cryst. 2015, 612, 110-116. doi:10.1080/15421406.2015.1030579

9. Konshina, E. A.; Galin, I. F.; Shcherbinin, D. P.; Gavrish, E. O. Liq. Cryst. 2014, 41, 1229-1234. doi:10.1080/02678292.2014.912764

10. Gavrish, E. O.; Galin, I. F.; Konshina, E. A.; Vakulin, D. A. Mol. Cryst. Liq. Cryst. 2015, 615, 50-56. doi:10.1080/15421406.2015.1066954

11. Galin, I. F.; Konshina, E. A. Mol. Cryst. Liq. Cryst. 2015, 615, 57-62. doi:10.1080/15421406.2015.1066955

12. Prodanov, M. F.; Pogorelova, N. V.; Kryshtal, A. P.; Klymchenko, A. S.; Mely, Y.; Semynozhenko, V. P.; Krivoshey, A. I.; Reznikov, Y. A.; Yarmolenko, S. N.; Goodby, J. W.; Vashchenko, V. V. Langmuir 2013, 29, 9301-9309. doi:10.1021/la401475b

13. Cîrtoaje, C.; Petrescu, E.; Stoian, V. Physica E 2015, 67, 23-27. doi:10.1016/j.physe.2014.11.004

14. Motoc, C.; Cirtoaje, C.; Stoica, A.; Stoian, V.; Albu, A. M. UPB Sci. Bull., Ser. A 2013, 75, 181-186.

15. Zakhlevnykh, A.N.; Lubnin, M. S.; Petrov, D. A. J. Magn. Magn. Mater. 2008, 431, 62-65. doi:10.1016/j.jmmm.2016.09.044

16. Ganea, C.; Manaila-Maximean, D. UPB Sci. Bull., Ser. A 2011, 73, 209-216.

17. Manaila-Maximean, D.; Rosu, C.; Yamamoto, T.; Yokoyama, H. Mol. Cryst. Liq. Cryst. 2004, 417, 215-226. doi:10.1080/15421400490478867

18. Fréedericksz, V.; Repiewa, A. Z. Phys. 1927, 42, 532-546. doi:10.1007/BF01397711

19. Fréedericksz, V.; Zolina, V. Trans. Faraday Soc. 1933, 29, 919-930. doi:10.1039/TF9332900919

20. Landau, L. D.; Lifshitz, E. M. Electrodynamics of Continuous Media; Pergamon Press: Oxford, United Kingdom, 1960.

21. Rapini, A.; Papoular, M. J. Phys. Colloques 1969, 30, 54-56. doi:10.1051/jphyscol:1969413

22. Burylov, S. V.; Raikher, Yu. L. J. Magn. Magn. Mater. 1993, 122 , 62-65. doi:10.1016/0304-8853(93)91040-E

\section{License and Terms}

This is an Open Access article under the terms of the Creative Commons Attribution License (http://creativecommons.org/licenses/by/4.0), which permits unrestricted use, distribution, and reproduction in any medium, provided the original work is properly cited.

The license is subject to the Beilstein Journal of Nanotechnology terms and conditions: (https://www.beilstein-journals.org/bjnano)

The definitive version of this article is the electronic one which can be found at:

doi:10.3762/bjnano.9.39 